\title{
A FRAMEWORK FOR SECURE DATA MANAGEMENT FOR MEDICAL DEVICES
}

\author{
Ibrahim Almazyad \\ Aakarsh Rao \\ Jerzy Rozenblit \\ Dept. of Electrical and Computer Engineering \\ University of Arizona \\ Tucson, AZ, USA \\ \{Almazyad, aakarshrao7, jerzyr\} @email.arizona.edu
}

\begin{abstract}
Data is a valuable currency that our modern world thrives upon. Security and privacy for data are becoming critical concerns, especially in healthcare where sensitive information is exchanged amongst the healthcare stakeholders. The protection of medical data is considerably more apparent and significant with guidelines such as HIPAA and FDA regulations in place. In this paper, we propose a methodology to ensure data security throughout the medical device life cycle, ranging from data initiation to data processing and data transmission. The goal is to facilitate communication between patients and doctors in a fast and secure manner. We propose an Adaptive Mode Selection (AMS) scheme to investigate security threats amongst system functions. A Priority-Queue Based (PQB) process is established to improve data management and data isolation within medical devices. Further, we propose an Adaptive Protocol Selection (APS) to enhance data transmission over the most appropriate communication protocol based on risk values identified by AMS. The combination of AMS, PQB and APS contributes towards delivering health services with continual secured data feeds and reduction in time of medical intervention.
\end{abstract}

Keywords: Implantable Medical Devices, Data Management, Medical Devices Security

\section{INTRODUCTION}

The realm of technology invades our society, as the footprint of digital devices continues to diversify with time and more services continue to migrate towards an online presence. Digital services play a pivotal role in the modern world. These digital services are expanding to healthcare with certain devices aiding towards well-being and livelihood. These devices, referred to as 'medical devices', have various purposes and functionalities. Some of these devices work as a replacement for a failed organ, while others support a weak organ. In any case, these medical devices carry out the required functionalities for the livelihood of an individual.

Medical devices such as a pacemaker, implantable cardiac defibrillator, or an insulin pump are referred to as an Implantable Medical Device (IMD) (Rushanan, Rubin, Kune, and Swanson 2014). In the United States alone, more than 25 million patients rely on IMDs to restore organ functionality, improve lifestyle quality and assist in essential functionality (Pope, Bouxsein, Manning, Hanna, et al. 2001). While these devices significantly improve patient healthcare, they also create a new set of security and privacy challenges throughout the healthcare ecosystem, that could directly affect safety. 
The Department of Health and Human Services (DHHS) reported 351 healthcare data breach incidents in the first half of 2017, equivalent to approximately 2 attacks per day (McLeod and Dolezel 2018). This entails addressing various security measures as IMDs collect data regarding a patient that is vital to perform the required treatment and provide an accurate assessment report about the patient's health. For example, a diabetic with an insulin pump has data collected about glucose levels in their blood, an average intake of insulin and battery life of the device. Doctors utilize such data to perform necessary re-configurations to the device and provide the needed care.

Our work will assist in improving communication between doctors and patients in a completely autonomous manner by developing a framework for secure data management. We look closely at the data life cycle among medical devices from the data initiation phase (how data is generated) to the transmission phase (how data is delivered to healthcare provider).

Security threats are taken into high consideration as we established an Adaptive Mode Selection (AMS) method. The AMS method assesses the system functionalities and blocks potential risks. This provides the medical device with the ability to have a united and lifetime assessment mechanism to enhance security. Moreover, the Priority-Queue Based (PQB) scheme improves managing data within life-critical systems. This provides an isolated environment, which limits data that is infiltrated and manipulated by attackers from spreading to other parts of the system. Additionally, a priority mechanism is established that improves processing and transmitting critical data. The Adaptive Protocol Selection (APS) looks at all aspects of the data transmission, prior to deciding the communication method and type of data transferred. Ultimately, (AMS), (PQB) and (APS) will facilitate delivering better health service quality by providing continuous secured data feeds which reduces intervention time when needed.

\section{BACKGROUND AND RELATED WORK}

IMDs assist with monitoring health status and providing treatment methods for patients. Diverse methods of communication have been researched by several works to overcome data security challenges. Researchers have introduced different novel methods to encrypt data prior to transmission. One example of this involves utilizing biometric values that uses Electrocardiograms (ECGs), blood glucose or other vitals to generate an encryption key (Rostami, Juels, and Koushanfar 2013, Hu, Cheng, Zhang, Wu, Liao, and Chen 2013, Venkatasubramanian, Banerjee, and Gupta 2009). As a result, we do not look at data encryption significantly within our research.

Another approach is using external wearable devices to provide authentication or mediate communication with IMDs (Denning, Fu, and Kohno 2008, Xu, Qin, Tan, Wang, and Li 2011). Islam and Yuce (Islam and Yuce 2016) present an example of this by utilizing the concept of continuous data feed. They aim to achieve accessible and secure data transmission through a physical band that acts as an intermediate for data transmission between healthcare providers and IMDs. A low-power, short-range communication system is used to access data from IMDs. Their work adopts the use of universal radio frequency 401 $406 \mathrm{MHz}$ dedicated to medical devices by the Federal Communications Commission (FCC) (Polka, Center, Lieberman, Friedman, Kissel, and Mueller 2010). Within a distance of two meters, the band collects data from IMDs and re-transmits them to the doctor. Although this concept improves a home-based station model, it introduces many challenges due to having the patient carry more devices that require additional care such as power and maintenance. Further, a single communication protocol is provided which is susceptible to signal loss due to an object interference or out of range distance.

Our goal is to enable autonomous communication between IMDs and healthcare providers. Specifically, we look more closely into insulin pumps. Due to the increasing demand from patients for monitoring glucose levels using phones, many insulin pump manufacturers have incorporated this feature. Thus we conducted 
a review for five common manufacturers and their insulin pump models equipped with continuous glucose monitoring sensors. We deduce the following disadvantages of such systems:

- Only glucose sensors are connected online. This provides limited access to data. The sensor only provides information about the current level of insulin in the blood. On the other hand, the pump itself provides considerably more information. This includes the amount of insulin administered "now and throughout the day", the method of delivering the insulin either "fast - bolus or slow basal", and the amount of insulin in the storage.

- The sensor is not fully autonomous. The sensor connects to the phone using Bluetooth which always requires an active phone. Furthermore, it requires running the application in the background to continuously receive the data.

- Gaps in data. If the connection to the phone is lost, data recovery is not possible in several sensors.

The Food and Drug Administration (FDA) in the United States encourages manufacturers to incorporate cybersecurity measures during the design life cycle of a device (FDA 2013). Towards this direction, a framework for secure design of medical devices has been discussed in (Rao, Carreón, Lysecky, and Rozenblit 2018, Rao, Rozenblit, Lysecky, and Sametinger 2017, Rao, Rozenblit, Lysecky, and Sametinger 2018). This work aims to address security threats through various design phases. The work proposed a method whereby medical device software is divided into functionalities that are placed into different modes of operation. Further, the design concept incorporated a run-time risk assessment that monitors potential threats in real-time. This enabled a mode switching methodology based on the risk assessed while maintaining a safe mode that includes life-critical functions. However, the secure framework design discussed in (Rao, Rozenblit, Lysecky, and Sametinger 2017) and (Rao, Rozenblit, Lysecky, and Sametinger 2018) focuses on software design for the device itself and does not consider safe data handling within the device and during transmission. Hence, in this work, we aim to expand the framework by improving the aspect of data management.

Our work in this paper will assist in improving communication between doctors and patients in a completely autonomous manner. At first, we look at how functions are selected to operate at each point in time before they generate data. The goal is to reduce the chance of infected functions operating on a medical device and increase system security. Further, we enhance the device's security by adopting data isolation methods that limit infected data accessed by hackers from spreading throughout the system. Hereafter, we add a mechanism that sorts the data depending on their importance to a patient's health. This assists in delivering patient information within the targeted time window. Moreover, adopting several well-known data transmission protocols will provide ease of use and prevent data gaps. Ultimately, this allows for delivering better health service quality through providing continuous secured data feeds which reduces intervention time when needed.

\section{DATA FLOW IN SOFTWARE SECURITY FRAMEWORK}

In order to understand how data flows within the medical device, a detailed examination of data life-cycle is conducted. This provides a closer perspective on how data flow within IMD systems, as shown in Figure 1.

- Threat detection and assessment: The security framework will assess each function for any possible threats. Based on a probabilistic model, it studies the behavior of the system and identifies any unexpected changes. As a result, each function is assigned a respective risk value as an indication of a possible threat (Rao, Carreón, Lysecky, and Rozenblit 2018). 


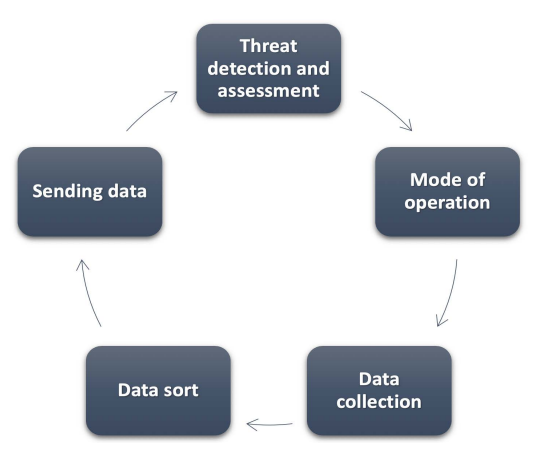

Figure 1: Data flow in a secure software framework model.

- Operating mode: After obtaining risk values from the initial phase, the middleware decides which mode is currently the safest for the device to operate in. A mode can consist of multiple functions that perform distinct tasks within the system.

- Data collection: During the data collection phase, the system collects distinct data from the functions that are allowed to operate within the specific operating mode. Examples of such data include the level of glucose in the body or the current heart rate of the patient. This data is then 4ed in queues until the data is processed or sent directly to the healthcare provider.

- Data sorting: After the data collection phase, the framework sorts each data packet based on a priority basis. Priority is assigned according to the relative importance of the function and the mode of origin as explained in 4.2.3. The priority value is ranging between 0 to 10 [0 - lowest, $10-$ highest]. As a result, data packets with higher priority values are presented at the top of the queue.

- Data transfer: After sorting the data packets in the queue, the systems sends this data to the healthcare provider through different channels. Data transmission is restricted to only functions that are whitelisted based on the mode of operation phase and current system status.

\section{THREAT DETECTION AND MODE SELECTION MECHANISM}

\subsection{Previous method to determine mode of operation}

Previous work conducted by (Rao, Rozenblit, Lysecky, and Sametinger 2017), assigned a cumulative risk for each mode of operation. For example, Mode 0 will only operate if the assigned risk value is between 0 and 20, Mode 1 will only operate if the risk value is between 20 and 40 and so forth. This method has several disadvantages, as it takes a general overview of the respective mode rather than a closer inspection of the mode and its functions. This results in some of the following shortcomings:

- Inactive functions - At the time of conducting an assessment, some functions may not be active therefore this may result in other active functions to incorrectly accumulate a higher risk value.

- No gradual shifts - As some functions may be severely compromised, a gradual shift of mode switching may not occur.

- No boundaries - With a system that consists of many functions and multi-levels, it is difficult to define the boundaries for each mode.

\subsection{Adaptive Mode Selection Mechanism}

The Adaptive Mode Selection (AMS) Mechanism aims to establish a dynamic connection between functions within each mode. This provides us with the ability to closely monitor risks assigned to each function. 
However, having different functions operating under different privileges could increase the complexity of the design. To address this, we divide the system functionalities into different groups based on their privileges such as criticality, importance, etc. Since each system may have different numbers of operating modes, no threshold is set for the number of groups as well.

We classify each of these function groups as a mode of operation. Functions classified as 'critical' are placed in Mode 0 and operates on a continuous basis, irrespective of mode switching. These groups of functions, named as modes, have the arrangement as shown in Figure 2

\begin{tabular}{|l|l|l|l|}
\hline Mode 0 & Function 1 & Function 2 & Function 3 \\
\hline \multicolumn{4}{|c|}{} \\
\hline Mode 1 & Function 4 & Function 5 & Function 6 \\
\hline \multicolumn{4}{|c|}{} \\
\hline Mode 2 & Function 7 & Function 8 & Function 9 \\
\hline
\end{tabular}

Figure 2: Functions assignment to each mode.

The layout illustrates three different modes of operation whereby each mode has its own privileges. In our system, Mode 0 carries the highest privilege level (critical) followed by Mode 1 (medium) and Mode 2 (low). As the number of modes increases, the privilege level for those respective modes decreases.

\subsubsection{Mode Inheritance}

As we established the placement of functions into different modes, our framework determines which modes are allowed to operate at a given point of time based on risk values. Each mode inherits functions from all modes with a higher priority level. This allows critical and important functions to operate, regardless of the current mode of operation. Further, in the case of an attack, the program isolates functions that are considered important in order to ensure maintaining basic healthcare functionalities. Figure 3 demonstrates this process and highlights which functions are allowed within each mode.

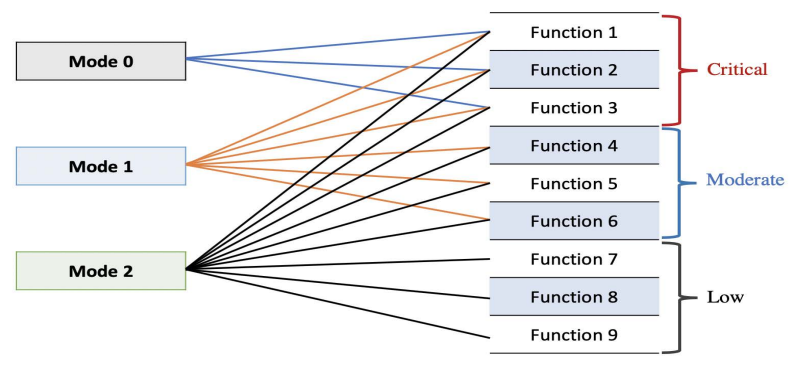

Figure 3: Mode Inheritance of Functions.

\subsubsection{Function Status}

During the operation of the system, functions within each mode can carry different states (i.e. status) based on the system schedule or patient request. In order to have an accurate representation of mode switching in our simulation, we take that into consideration. Whilst the functions are active, the system continues to assess the risk ensuring that they are within the assigned limit. However, as functions begin to transition into an idle phase, the framework eliminates them from risk calculation to ensure the accuracy of the mode selection mechanism. 


\subsubsection{Function Risk and Threshold}

Each function within the system could introduce a potential threat to a patient. It could be a critical function related to the device such as an insulin monitoring method or a trivial function that changes the alarm tone of a particular device. In either case, these functions could introduce threats to implantable medical devices (IMDs).

In order to have an accurate understanding of the overall risk associated with the system, we examined each function individually. This opens up a new possibility to understand the safety of each mode and the safety of the overall system as well. Prior to allowing functions to be activated, two factors are considered. The first factor is the 'function risk' - this is given by a probabilistic model that examines a function at any point of time and provides an estimate of a potential threat. The model assigns a value ranging from 0 (a very high probabilistic risk value) to 10 (a very low probabilistic risk value) (Rao, Carreón, Lysecky, and Rozenblit 2018). The second factor is a threshold that provides a risk limit which should not be exceeded at any point of time. This value identifies the boundaries within which the function must operate. If the risk values for each function is within the allowable limits, that particular mode is selected. On the contrary, if the risk values for each function is above the determined threshold value, the system is posed with two options:

1. If the function has a low priority or considered as an unimportant function, then it can be deactivated alone.

2. If the function has a high priority or considered critical to the patient, then the system switches to a safer mode that can accommodate the risk value of the function.

Throughout this process, the system continues to identify a stable mode that allows function(s) to operate without introducing risks to the patient. In every iteration, the system continues to check risk and threshold values to ensure functions are within safe limits. The overall algorithm is shown in Figure 4.

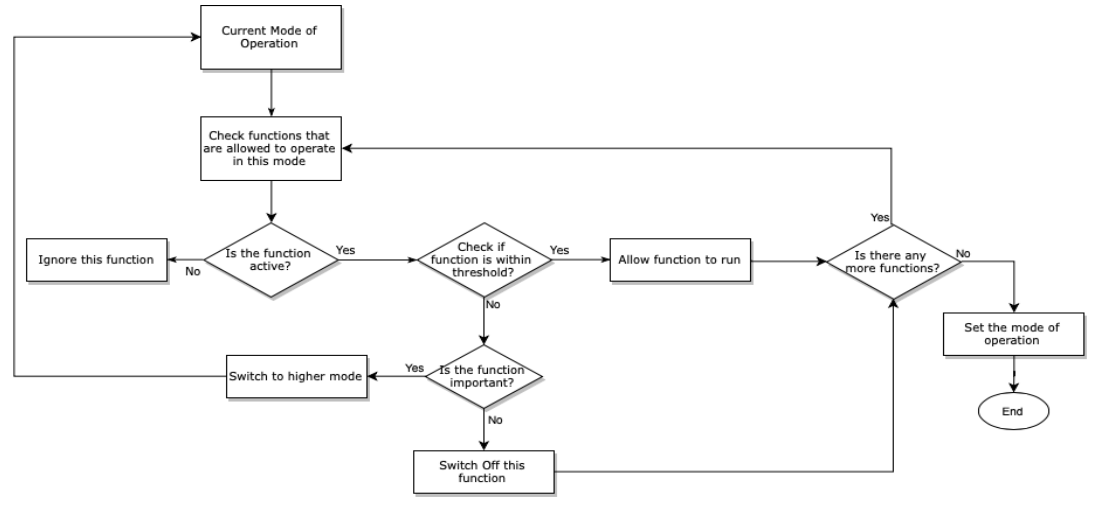

Figure 4: Adaptive Mode Selection Algorithm.

In order for a function to be activated, the risk value should be above the identified threshold level, as represented by the blue region of Figure 5 (a). The value of threshold is dynamically assigned to each function based on the following:

- Function ID $(n)$ : represent a unique identification number for a function

- Mode of Origin $(\alpha)$ : represents the initial mode where the function was placed. This is represented in Figure 2

- Mode of operation $(\beta)$ : represents the current mode of operation in the system 


\section{Almazyad, Rao and Rozenblit}
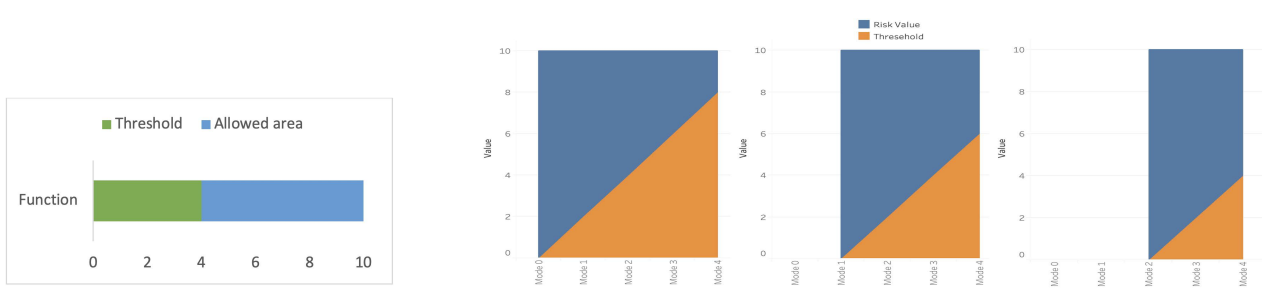

Figure 5: (a) Function risk and threshold. (b) Risk tolerance in respect to mode of origin.

- Increment factor $(x)$ : represents the incremental value of the threshold

$$
\text { Threshold for } n=x *(\beta-\alpha) \text {. }
$$

The equation (1) adjusts the threshold for each function irrespective of its mode of origin $(\alpha)$. It provides a gradual increment for the threshold, based on the difference between mode of operation and origin $(\beta-\alpha)$. The incremental value $(x)$ can be adjusted based on the number of levels available within the system. Based on our experiments, we utilized a factor of two $(x=2)$ for our simulation case study.

The value of threshold is dynamically assigned to each function. As functions migrate towards operating in a mode distant from the mode of origin, they tend to have a lesser tolerance to risk. In return, the threshold value assists in detecting threats at early stages to reduce the possibility of having a severe compromise. This approach is demonstrated in Figure 5 (b).

In the event that a threat is detected within a certain mode, the framework utilizes a "zip ties" methodology to protect other modes. In this method, we will be able to shift to modes of operation with a higher priority only. With this 'lock' mechanism approach, the system switches to a safer mode that can accommodate the detected threat and associated risk(s). The system continues to operate in this manner until it identifies the safest mode of operation. With this downgrade approach, the framework locks the current mode's functions and associated data to prevent any infected data from transitioning between modes. The system continues to operate without the need for unnecessary functions. This 'lock' mechanism can be lifted after a specialist manually investigates the locked mode(s) to ensure their safety.

We built a simulation of our framework to showcase the functioning of our algorithm. As shown in Figure 6 , the system maintained a steady performance while operating in Mode 2 for about 3400 cycles. After a function's risk increases to 5, less than the assigned threshold limit (6), the framework successfully transitions from Mode 2 to Mode 1 to accommodate the current risk. That particular function that posed a risk is disabled to eliminate potential future threats.

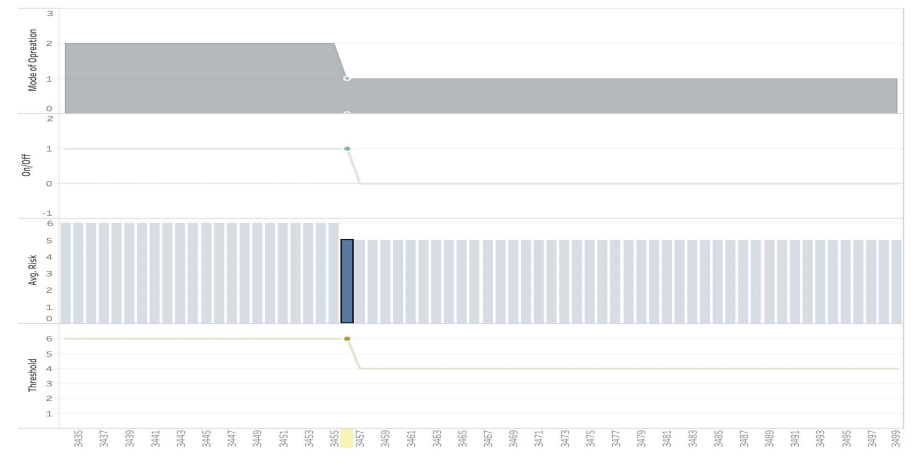

Figure 6: Adaptive Mode Switching Implementation. 


\section{SECURE DATA MANAGEMENT}

Managing a large number of processes within a system requires a scheduling mechanism. In our system, we identify multiple factors that affect medical device data that include: data priority, data waiting time and data security. As a result, we propose a multi-level Priority-Queue Based (PQB) system for the medical device middleware. Multi-level queueing is the best fit for scenarios where tasks are divided into groups based on a common feature such as process type, CPU time, and memory size (Silberschatz, Gagne, and Galvin 2018). For such an algorithm, the system has several 'n' queues depending on the number of groups the system tasks are divided into. Processes among each queue will have a separate scheduling algorithm, such as first-in-first-out and Round Robin algorithms, defining the queueing mechanism for the tasks. Each queue is then assigned a priority value to determine the order in which queues are processed. A high priority queue needs to be empty before moving to the following queue. Implementing this queuing system enables the handling of data management securely and promptly.

Storing large amounts of information necessitates an efficient method that responds to data demand dynamically (Overmars 1987). Devices that have a limited amount of resources such as IMDs, require a proper memory management technique to be adopted. Dynamic data structures allow the use of device memory without predefining the size of the structure. When a structure is needed, it reallocates the needed space and then attaches it using pointers. The overall view of how the queuing system works in a secure middleware of a medical device is shown in Figure 7.

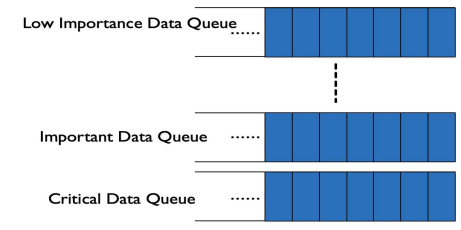

Figure 7: Multi Level Queues.

Each queue adapts Priority-Queue Based (PQB) approach to store data incoming from functions with a certain level of importance. This method allows for functions with the same privileges to share the same part of the memory. As a result, we can achieve the following important objectives:

- Data with higher privileges will have a faster processing time. Since a multi-level queuing system allows us to separate the data into different queues based on their privileges, it will select queues with a high priority to be processed before any remaining queues. This ensures that important and critical data are transmitted promptly when needed.

- Data breach countermeasures. Breaches in patient data are a critical threat that healthcare providers continue to encounter. Breaches may include deletion or modification of the content of a file. To protect our system from data breaches, we implement the multi-level queue method through randomizing memory locations to increase security measures for the medical device. Further, as we classify data queues based on their priority, this process ensures withholding compromised data to protect other remaining data queues. For example, if a low priority data queue is compromised, it does not affect high priority data queues that provide critical tasks/functions to a patient.

\subsection{Task Scheduler}

The Task scheduler handles all data requests within IMDs. Data can be accessed by functions within the device for purposes such as local processing or delivering it to healthcare providers for further analysis and monitoring. In this paper, we focus on data that is transmitted to doctors and healthcare practitioners. The 
Adaptive Protocol Selection (APS) decides which data is transmitted and what medium is used, without endangering the security of the device. This process is based on two factors: system status and mode of operation, as represented in Figure 8.

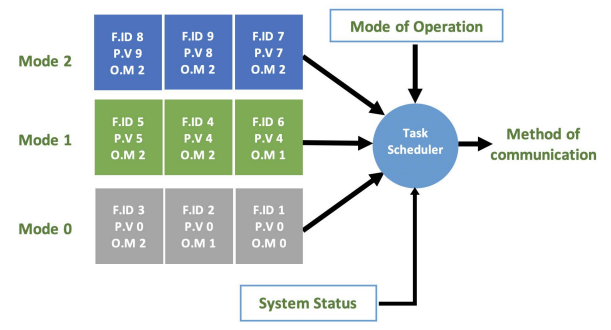

Figure 8: Adaptive Protocol Selection.

\subsubsection{System status}

System status represents the overall activity of the medical device at a given moment.

- Active Status: During the active status state, the system consists of operating function(s) that perform certain tasks such as user request, system request and sensor request. During this state, it is important to reduce the processing time to perform these functions as they are crucial to a patient's life.

- Idle Status: During the idle status, the system operates with no important functions at a given point in time. Our goal is to exploit the idle time to transmit data without affecting the performance of the system.

\subsubsection{Mode of operation}

As explained earlier in the AMS mechanism, we assess risk values for each function over time. As the system enters Idle Status, we have an assessment of potential risk the system might encounter from each function before allowing it to run. Based on these values, this mechanism provides an optimal mode of operation for the system to operate in. The communication method is selected as follows:

- Mode 0: Allows us to transfer only critical data through a secure connection. Only life-critical functions can operate to eliminate risks of compromising a patient's heath. These functions are isolated from the external environment to increase security measures within the device. When the data is required to be transferred, a secure connection is selected, as shown in Figure 9 (c). This could be done through using a secure home station where data is securely transferred, or by visiting the doctor's office and taking readings directly from the device.

- Mode 1: Allows critical and moderate data to be transferred using Bluetooth, as shown in Figure 9 (b). This enables a connection between smartphones and medical devices within a short-range. A smartphone is used as a way of communication to carry data packets to healthcare providers. This eases the method of communication as it integrates very well with common smartphones. Further, smartphones are used as a shield to protect IMDs from potential cyber threats.

- Mode 2: Allows data incoming from all three modes within the system to be sent through Wi-Fi, as shown in Figure 9 (a). This enables the device to operate autonomously through managing and transferring data. Further, it initiates a direct line of communication between IMDs and healthcare 


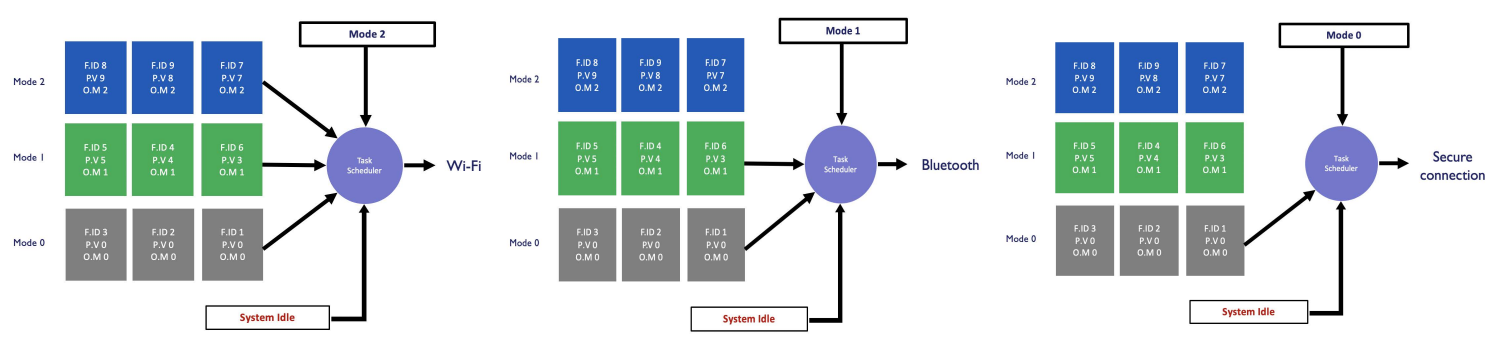

Figure 9: Data Transmission within (a) Mode 2, (b) Mode 1 and (c) Mode 0.

providers without additional work from the patient. This facilitates providing a continuous feedback loop in a secure and user-friendly manner.

\subsection{Performance Analysis}

In order to measure the performance of a queue and compare it to other queues, we add variables to each data packet that helps in monitoring data packet processing within each queue. These variables are as follows:

- Arrival Time (AT): represents the time when the packet is placed in the queue

- Burst Time (BT): represents an estimate of how long it takes to process this packet

Adding these values assists in gaining further understanding of the queueing system performance by conducting the following calculations:

- Completion Time (CT): represents the time when the packet is processed and sent to the healthcare provider

- Turn Around Time (TAT): represents the period of time from the packet processing time until its completion time, as shown in equation (2)

$$
T A T=C T-T A T .
$$

- Waiting Time (WT): Represents the amount of time that the packet has to wait until it is processed. This is the most important measurement because it provides the amount of delay that is experienced by the critical packets, as shown in equation (3)

$$
W T=T A T-B T .
$$

Now, we calculate the overall performance of the system by averaging the waiting time for each queue, equation (4).

$$
\text { Avg } W T=(\text { Sum } W T / \text { Number of Packets }) \text {. }
$$

Queue 0 has functions that are critical to a patient's life. For this reason, we want to deliver the data as fast as possible in order to keep healthcare providers informed with the most recent critical data. Moreover, as we start moving towards less critical data, the time delay becomes more tolerable. The result of the average waiting time for each queue in our case study is shown in Figure 10. Queue 0, with the most critical data, has an average waiting time of 30 milliseconds. On the other hand, Queue 2, which contains non-critical data, has an average waiting time of 137 milliseconds. This shows how the system provides a higher priority for critical data as it had a processing time that is faster than non-critical data. 


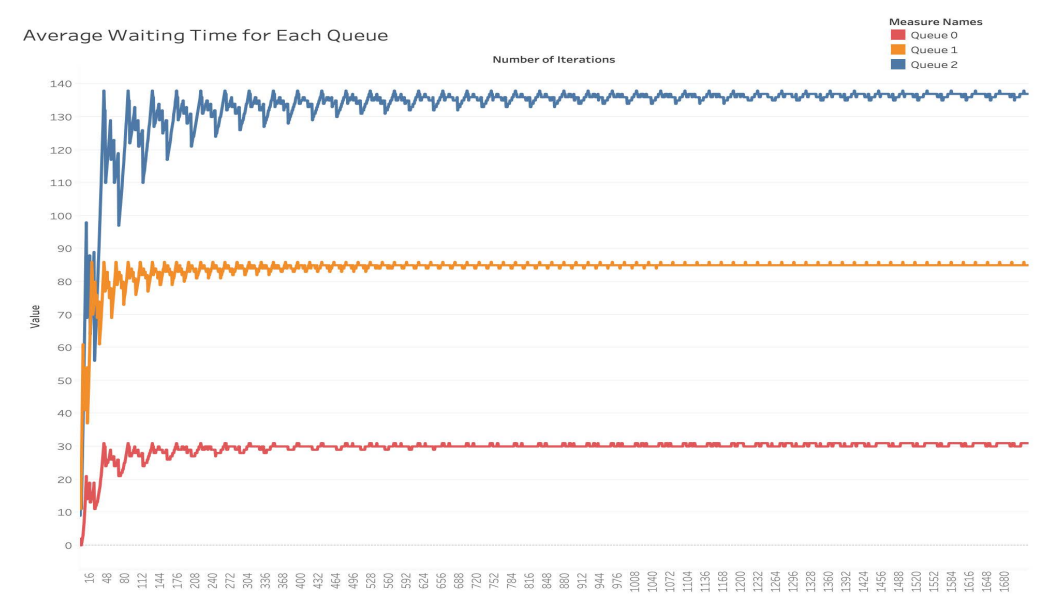

Figure 10: Average Waiting Time for Each Queue.

\section{CONCLUSION AND FUTURE WORK}

Our goal in this paper is to deliver data with minimal effort through establishing different modes of communication. With an increasing number of cybersecurity threats on the horizon, several challenges need to be overcome to achieve such a goal. Our approach aims to ensure data security throughout each phase within the medical device, ranging from data initiation, data processing and data transmission. Before deploying our framework into medical devices, several areas need further consideration such as low overhead encryption mechanism and power optimization. As the IMDs have limited resources, further analysis is needed to ensure proper utilization of resources. Ultimately, the framework will enhance the communication that occurs between a patient and their doctor. In doing so, it will reduce the time taken for medical intervention and therefore improves the quality of healthcare services.

\section{REFERENCES}

Denning, T., K. Fu, and T. Kohno. 2008. "Absence Makes the Heart Grow Fonder: New Directions for Implantable Medical Device Security.”. In HotSec.

FDA 2013. "Content of premarket submissions for management of cybersecurity in medical devices: Draft guidance for industry and Food and Drug Administration staff.".

Hu, C., X. Cheng, F. Zhang, D. Wu, X. Liao, and D. Chen. 2013. "OPFKA: Secure and efficient orderedphysiological-feature-based key agreement for wireless body area networks". In 2013 Proceedings IEEE INFOCOM, pp. 2274-2282. IEEE.

Islam, M. N., and M. R. Yuce. 2016. "Review of medical implant communication system (MICS) band and network". Ict Express vol. 2 (4), pp. 188-194.

McLeod, Alexander and Dolezel, Diane 2018. "Cyber-analytics: Modeling factors associated with healthcare data breaches".

Overmars, M. H. 1987. The design of dynamic data structures, Volume 156. Springer Science \& Business Media.

Polka, M. M., O. P. Center, R. J. Lieberman, S. C. Friedman, J. M. Kissel, and C. Mueller. 2010. "Before the Federal Communications Commission Washington, DC 20554".

Pope, A., P. Bouxsein, F. J. Manning, K. E. Hanna et al. 2001. Innovation and invention in medical devices: workshop summary. National Academies Press. 
Rao, A., N. Carreón, R. Lysecky, and J. Rozenblit. 2018, January. "Probabilistic Threat Detection for Risk Management in Cyber-physical Medical Systems". IEEE Software vol. 35 (1), pp. 38-43.

Rao, A., J. Rozenblit, R. Lysecky, and J. Sametinger. 2017. "Composite risk modeling for automated threat mitigation in medical devices". In Proceedings of the Symposium on Modeling and Simulation in Medicine, pp. 9. Society for Computer Simulation International.

Rao, A., J. Rozenblit, R. Lysecky, and J. Sametinger. 2018. "Trustworthy multi-modal framework for lifecritical systems security". In Proceedings of the Annual Simulation Symposium, pp. 17. Society for Computer Simulation International.

Rostami, M., A. Juels, and F. Koushanfar. 2013. "Heart-to-heart (H2H): authentication for implanted medical devices". In Proceedings of the 2013 ACM SIGSAC conference on Computer \& communications security, pp. 1099-1112. ACM.

Rushanan, M., A. D. Rubin, D. F. Kune, and C. M. Swanson. 2014. "Sok: Security and privacy in implantable medical devices and body area networks". In 2014 IEEE Symposium on Security and Privacy, pp. 524-539. IEEE.

Silberschatz, A., G. Gagne, and P. B. Galvin. 2018. Operating system concepts. Wiley.

Venkatasubramanian, K. K., A. Banerjee, and S. K. S. Gupta. 2009. "PSKA: Usable and secure key agreement scheme for body area networks". IEEE Transactions on Information Technology in Biomedicine vol. 14 (1), pp. 60-68.

Xu, F., Z. Qin, C. C. Tan, B. Wang, and Q. Li. 2011. "IMDGuard: Securing implantable medical devices with the external wearable guardian”. In 2011 Proceedings IEEE INFOCOM, pp. 1862-1870. IEEE.

\section{AUTHOR BIOGRAPHIES}

IBRAHIM ALMAZYAD is currently a lecturer at Qassim University in Saudi Arabia. He used to work in the Model-Based Design Laboratory at the University of Arizona. He works on incorporating data security within life-critical frameworks such as medical devices, with a focus on enhancing communication between patient and healthcare providers. He received his Masters in Electrical and Computer Engineering from the University of Arizona in 2019. He received his B.S in Computer Engineering from Qassim University, Saudi Arabia in 2015. His email is almazyad@email.arizona.edu.

AAKARSH RAO is currently a PhD student in the Electrical and Computer Engineering Department at the University of Arizona. He works in the Model-Based Design Laboratory on medical device security and design. He received his Masters in January 2015 from the same university and B.S in Electronics and Communication from M.S. Ramaiah Institute of Technology, India in 2009. His email is aakarshrao7@email.arizona.edu.

JERZY ROZENBLIT is a University Distinguished Professor, Raymond J. Oglethorpe Endowed Chair in the Electrical and Computer Engineering (ECE) Department, with a joint appointment as Professor of Surgery in the College of Medicine at the University of Arizona. During his tenure at the University of Arizona, he established the Model-Based Design Laboratory with major projects in design and analysis of complex, computer-based systems, hardware/software codesign, and simulation modeling. He presently serves as Director of the Life-Critical Computing Systems Initiative, a research enterprise intended to improve the reliability and safety of technology in healthcare and life-critical applications. His email address is jerzyr@email.arizona.edu. 